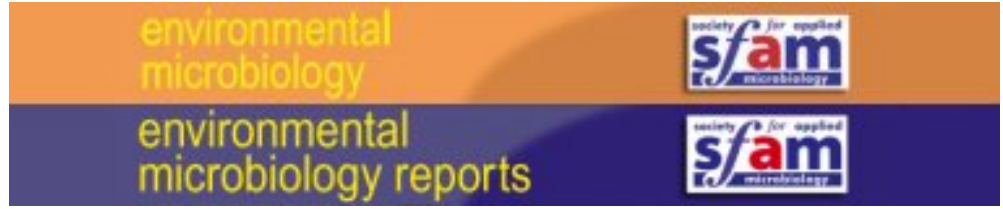

\title{
The zoospores of the thraustochytrid Aurantiochytrium limacinum: transcriptional reprogramming and lipid metabolism associated to their specific functions.
}

\begin{tabular}{|r|l|}
\hline Journal: & Environmental Microbiology and Environmental Microbiology Reports \\
\hline Manuscript ID & EMI-2019-1652.R2 \\
\hline Journal: & Environmental Microbiology \\
\hline Manuscript Type: & EMI - Research article \\
\hline Date Submitted by the & O4-Mar-2020 \\
\hline Complete List of Authors: & $\begin{array}{l}\text { Dellero, Younès; Institut de Biosciences et Biotechnologies de Grenoble } \\
\text { (BIG), Laboratoire Physiologie Cellulaire \& Végétale (LPCV) } \\
\text { Maës, Cécile; Institut de Biosciences et Biotechnologies de Grenoble } \\
\text { (BIG), Laboratoire Physiologie Cellulaire \& Végétale (LPCV) } \\
\text { MORABITO, Christian; Institut de Biosciences et Biotechnologies de } \\
\text { Grenoble (BIG), Laboratoire Physiologie Cellulaire \& Végétale (LPCV) } \\
\text { Schuler, Martin; Institut de Biosciences et Biotechnologies de Grenoble } \\
\text { (BIG), Laboratoire Physiologie Cellulaire \& Végétale (LPCV) } \\
\text { Bournaud, Caroline; Institut de Biosciences et Biotechnologies de } \\
\text { Grenoble (BIG), Laboratoire Physiologie Cellulaire \& Végétale (LPCV) } \\
\text { Aiese Cigliano, Riccardo; Sequentia Biotech } \\
\text { Maréchal, Eric; Institut de Biosciences et Biotechnologies de Grenoble } \\
\text { (BIG), Laboratoire Physiologie Cellulaire \& Végétale (LPCV) } \\
\text { Amato, Alberto; Interdisciplinary Research Institute of Grenoble, LPCV } \\
\text { Rébeillé, Fabrice; Institut de Biosciences et Biotechnologies de Grenoble } \\
\text { (BIG), Laboratoire Physiologie Cellulaire \& Végétale (LPCV) }\end{array}$ \\
\hline $\begin{array}{l}\text { Thraustochytrids, Zoospores, RNA-sequencing, Fatty acids, PUFA } \\
\text { Kynthase, w3-docosahexaenoic acid, Lipid metabolism, algae }\end{array}$ \\
\hline
\end{tabular}

\section{SCHOLARONE Manuscripts}


1 The zoospores of the thraustochytrid Aurantiochytrium limacinum: transcriptional reprogramming

2 and lipid metabolism associated to their specific functions.

3

4 Younès Dellero ${ }^{1,3^{*}}$, Cécile Maës $^{{ }^{*}}{ }^{*}$, Christian Morabito ${ }^{1^{*}}$, Martin Schuler ${ }^{1}$, Caroline Bournaud ${ }^{1}$, Riccardo

5 Aiese Cigliano ${ }^{2}$, Eric Maréchal ${ }^{1}$, Alberto Amato ${ }^{1 \#}$ and Fabrice Rébeillé ${ }^{1 \#}$

$6{ }^{1}$ Laboratoire de Physiologie Cellulaire Végétale, Université Grenoble Alpes, CEA, CNRS, INRA; IRIG-

$7 \quad$ LPCV 38054, Grenoble Cedex 9, France

$8 \quad 2$ Sequentia Biotech Campus UAB, Edifici Eureka Av. de Can Domènech s/n 08193 Bellaterra

9 (Cerdanyola del Vallès), Spain

${ }^{3}$ Present address: Institute of Genetic, Environment and Plant Protection, UMR 1349 IGEPP

11 INRA/Agrocampus Ouest Rennes/Université Rennes 1, Domaine de la Motte, BP35327, 35653 Le

12 Rheu cedex, France

$14 \quad$ \#Corresponding authors

15 *These authors contributed equally to this work

16 Email addresses: fabrice.rebeille@cea.fr (F. Rébeillé),. alberto.amato@cea.fr (A. Amato) 


\section{Abstract}

Aurantiochytrium limacinum (Thraustochytriaceae, class Labyrinthulomycetes) is a marine

Stramenopile and a pioneering mangrove decomposer. Its life cycle involves a non-motile stage and zoospore production. We observed that the composition of the medium, the presence of amino acids in particular, affects the release of zoospores. Two opposite conditions were defined, one with a cell population mainly composed of zoospores and another one with almost only non-motile cells. In silico allelic frequency analysis and flow cytometry suggest that zoospores and non-motile cells share the same ploidy level and are diploid. Through a RNA-seq approach, the transcriptional reprogramming accompanying the formation of zoospores was investigated, with a particular focus on their lipid metabolism. Based on a differential expression analysis, zoospores are characterized by high motility, very active signal transduction, an arrest of the cell division, a low amino acid metabolism and low glycolysis. Focusing on lipid metabolism, genes involved in lipase activities and peroxisomal $\beta$-oxidation are up-regulated. qRT-PCR of selected lipid genes and lipid analyses during the life span of zoospores confirmed our observations. These results highlight the importance of the lipid dynamics in zoospores and show the metabolic processes required to use these energy-dense molecules as fuel for zoospore survival during their quest of new territories.

Key words: Fatty acids; GPCR pathway; Lipid metabolism; PUFA synthase; RNA-sequencing; Signal transduction; Thraustochytrids; Zoospores; w3-docosahexaenoic acid (DHA). 


\section{Introduction}

Mangrove forest environments sustain a composite ecosystem (Isa et al., 2017), with a food web primed by decomposers (Demopoulos et al., 2007). Thraustochytrids are osmo-saprotrophic protists belonging to the phylum Heterokonta, class Labyrinthulomycetes (Cavalier-Smith et al., 1995) and are among the first organisms that colonize and decompose a fallen mangrove leaf (Raghukumar et al., 1994). The life cycle of thraustochytrids is characterized by vegetative reproduction and a motile stage, the zoospores (Honda et al., 1998, Iwata et al., 2017, Morabito et al., 2019). The biflagellated zoospores released from sporangia are essential to colonize new environments and propagate the population. Thraustochytrids are nutritionally valuable preys for microzoo- and zoo-plankton, especially because of their high content in very long chain polyunsaturated fatty acids (VLC-PUFAs) (Kimura and Naganuma, 2001 and references therein, Dellero et al., 2018a). Furthermore, the coastal zone they inhabit is the interface between the land-fixed and the ocean captured carbon, the socalled 'Blue Carbon' (Nelleman et al., 2009). Therefore, thraustochytrids may play a pivotal role in the 'mycoloop', a recently described energy pathway based on chytrid zoospores (Kagami et al., 2014). Through the mycoloop refractory or inaccessible carbon can be transferred to the zooplankton (Kagami et al., 2007).

Although several organisms living in aquatic environments, such as algae, fungi, oomycetes and bacteria are able to produce zoospores (Agrawal, 2012), the literature concerning their metabolism and physiology is quite scarce. Most of the available studies concerning eukaryotic zoospores were carried out on oomycetes because several oomycetes, such as Phytophthora infestans, are plant pathogens and zoospores initiate the infectious interaction with the hosts (Hardham, 2007). This feature has fostered research to decipher the physiology of infective zoospores, with the aim to prevent infection, improve cultivation and reduce crop lost. The release of zoospores from sporangia may depend on several parameters such as light, nutrients, temperature shifts or intracellular pH variations (Hyde et al., 1991, Suzaki et al., 1996, Agrawal, 2012). Once produced, zoospores sense and reach new territories via phototaxis, electrotaxis or chemotaxis (Agrawal, 2012, Swafford and 
Oakley, 2017). In the last decade, several studies on oomycetes focused on the identification of infection-related factors using transcriptomics approaches (Judelson et al., 2008; Chen et al., 2013, 2014; Sun et al., 2017). A panel of predicted genes, including transcription factors, protein kinases, putative signaling proteins, ion channel, or calcium binding proteins were suggested to be involved in the formation, survival and physiology of zoospores (Tani et al., 2004; Ah-Fong et al., 2017). In contrast with oomycetes, little information is available about the zoospore physiology and metabolism of non-infective thraustochytrids. Recently, thraustochytrids have attracted industrial interest for their ability to synthesize high levels of VLC-PUFAs, in particular docosahexaenoic acid (DHA, 22:6), an essential compound for human health (Simopoulo, 2008). Indeed, thraustochytrids have the unique feature to synthesize FA via two enzymatic systems; the classical fatty acid synthase (FAS) involved in the synthesis of relatively short saturated FA chains (16 to 18 carbons) and the PUFA synthase involved in the synthesis of longer unsaturated chains, such as DHA (Morabito et al., 2019). The biotechnological interest towards thraustochytrids has directed efforts to unravel their physiology and metabolism, with the main aim to enhance lipid production (Raghukumar, 2008; Taoka et al., 2009; Aasen et al., 2016). This has prompted genomic and transcriptomic studies (Ma et al., 2015; Liu et al., 2016; Bi Z.-Q. et al., 2018; Iwasaka et al., 2018; Liang et al., 2018; Seddiki et al., 2018) which provide today new databases for metabolic engineering purposes and for ecophysiological understanding as well.

In a recent work, we investigated the ecophysiology of Aurantiochytrium limacinum zoospores and the triggers for their formation, settlement and maturation. Zoospores are released upon a transfer to a fresh nutrient-poor medium. They can swim for several days, relying on their energy storage for cell survival and motility (Dellero et al., 2018a). These observations raise questions about the nutritional factors that trigger a shift from a sedentary to a motile state, and about the associated transcriptional reprogramming and metabolic changes. Here we further describe the role of the external medium in the release of zoospores and depict the metabolic and transcriptional signatures 
90 associated with the vegetative-to-zoospore transition, with an emphasis on lipid metabolism. Our

91 results reveal that such a transition implies profound transcriptional and metabolic rearrangements. 


\section{Results}

Zoospore release

As previously shown (Dellero et al., 2018a), in a rich medium (R) containing glucose and yeast extract (YE) less than $3 \%$ zoospores were observed at best (D1), whereas in a poor medium (P), in which the glucose and YE concentrations were reduced to 1/40 (Supplementary Table 1), zoospores represented the main cell type (Table 1). A different medium, named $\mathrm{R} 2$, was produced in order to rule out the possibility that one or several components of the YE might repress zoosporulation. In the R2 medium YE was replaced by nitrate and phosphate solutions at concentrations comparable to those found in YE (Supplementary Table 1). A six-day-old R-grown culture, i.e. which did not contain any zoospores, was used as inoculum. During the first 24 hours in R2, zoospores were massively produced and represented $92 \%$ of the population (Table 1). A high zoospores/non-motile cells ratio was maintained until day 4 (D4). Thereafter, however, zoospore fraction decreased to nil and nonmotile cells dominated the population. It can be concluded that one of the YE components may impair the release of zoospores.

In order to discriminate whether amino acids (the main source of nitrogen in R) hamper zoosporulation, nitrate was removed from R2 and replaced by a cocktail of amino acids in concentrations that mimic YE (R3 medium, Supplementary Table 1). The zoospore/non-motile cells ratio in R3 was comparable to that recorded in R, i.e. very few zoospores were observed. Inversely, when amino acids were added in a P culture containing almost exclusively zoospores, the non-motile cell occurrence increased at D4 (i.e. 48 hours after the addition of amino acids). At D6, a $3.4 \%$ zoospore/non-motile cells ratio was recorded. Altogether, these results highlight a potential role of amino acids in controlling the initiation/maturation of the motile stage of $A$. limacinum.

\section{Ploidy level of the zoospores and non-motile cells}

A variant calling analysis was performed by mapping the transcriptomes of non-motile cells and zoospores produced in the present work against the Aurantiochytrium limacinum ATCC ${ }^{\circledR}$ MYA1381 ${ }^{\mathrm{TM}}$ reference genome. 38110 (Supplementary file 1) and 53181 (Supplementary file 2) variants were 
identified in non-motile cells and zoospores, respectively. Single Nucleotide Polymorphisms (SNPs)

represented about $94 \%$ of the variants in both groups. A vast majority of the variants (64 \% and $63 \%$ of total variants for non-motile and zoospores, respectively; Supplementary Files 1 and 2) were identified as alternative genotypes, i.e. all the reads in the transcriptome of non-motile cells and zoospores presented the same sequence, different from that in the reference genome. Such sites can be classified as intraspecific polymorphisms between the Aurantiochytrium limacinum ATCC $^{\circledR}$ MYA1381 ${ }^{\mathrm{TM}}$ used as reference and $A$. limacinum CCAP 4062/1 strain. In order to estimate the ploidy level of zoospores and non-motile cells, allele frequency distributions were calculated (Fig. 1A). The peak of 'homozygous variants' (allele frequency $=100$ ) on the right-hand side of the plots (Fig. 1A) actually identifies the intraspecific polymorphic sites described above. Noteworthy, 13829 (36 \%) and 19854 (37 \%) variants were classified as heterozygous (0/1 or 1/0 genotype, Supplementary Files 1 and 2) in non-motile cells and zoospores, respectively. The allele density (y-axis in Fig. 1A) is an indication of the number of reads bearing one or the other allele. A Mann Whitney Test was performed to compare the distribution of the allele frequencies of non-motile cells and zoospores and the difference resulted not significant $(p=0.35)$. The portion of the allele frequency curve of the heterozygous variants only (Fig. 1A) is centered on 50 in both samples, therefore, it can be stated that zoospores and non-motile cells share the same ploidy level and that they are both diploid. In order to validate the latter result, flow cytometry was used to measure the quantity of DNA in zoospores vs. non-motile cells.

In flow cytometry, light scatter analysis of a fast growing population in R showed a large panel of cell sizes and internal complexity (granulometry) (Fig. 1B). The fluorescence pattern of propidium iodide (PI) of R-grown cells displayed a main peak at an arbitrary value of 280 and a second one representing cells containing about twice as much DNA as the cells forming the first peak (Fig. 1C).

141 The right-hand side of the figure indicates the presence of cells containing even more DNA. Assuming

142 that $A$. limacinum is diploid (as suggested by allele frequency analysis), the first peak would represent 143 diploid mononuleated cells and the second either mononucleated cells in G2 phase or binucleated 
cells. Because of the presence of multinucleated cells, the interpretation of the following peaks is cumbersome. Each peak could represent either a cell population with n nuclei in G2 phase or cells with $2 \mathrm{n}$ nuclei in G1 phase. On the contrary, zoospore size and granulometry was more uniform (Fig. 1D). PI fluorescence (Fig. 1E) showed a main and relatively sharp peak and a second small peak representing cells with twice as much DNA. By comparing Figures $1 \mathrm{C}$ and $1 \mathrm{E}$, it is clear that zoospores did not contain half the DNA than non-motile cells. These results strongly suggest that zoospores are diploid, corroborating the allele frequency analysis (Fig. 1A).

\section{Genome wide expression and transcriptional footprints in zoospores}

A differential expression analysis based on RNA-seq data showed that in zoospores 4280 and 3330 genes were up- and down-regulated (cut-off arbitrarily set at $\log _{2} \mathrm{FC}=1$ ), respectively

(Supplementary Table 2). 7610 genes represent about half of the whole genome. RNA-seq differential gene expression analysis was validated by qRT-PCR of 22 selected genes (Supplementary Table 3) on RNA extracted from a completely independent experiment carried out exactly as described for RNAseq. The Pearson's correlation coefficient $(r=0.93)$ showed a strong positive linear correlation ( $p$ value $<10^{-5}$ ) between qRT-PCR and RNA-seq analyses. The strong linear correlation was coupled with a strong monotonic relation between the two sets of results by Spearman's Rho $\left(r_{\mathrm{S}}=0.88, p\right.$-value $=$ 0.05).

From a Gene Ontology Enrichment Analysis (GOEA) (Supplementary Fig. 1), most of the significantly enriched up-regulated GO terms were 'signal transduction' (GO:0007165) and several descendants like 'G-protein coupled receptor signaling pathway' (GO:0007186), or 'signal transducer activity' (GO:0097677) which groups mainly transcription factors. This is indicative of an increased ability of the zoospores to sense and react to their external environment. Zoospores are biflagellated cells and some of the highly enriched GO terms among the up-regulated genes are, not surprisingly, 'microtubule associated complex' (GO:0005875), 'dynein complex' (GO:0030286), 'motor activity' (GO:0003774). Among the down-regulated genes, 'translation' (GO:0006412), 'aminoacyl-tRNA ligase 
activity' (GO:0004812), 'structural constituent of ribosome' (GO:0003735) were the most enriched GO terms, suggesting an arrest of the cell division and a lower turnover of transcripts.

\section{Signal transduction}

Genes involved in signal transduction and signaling cascades were strongly expressed in zoospores (Fig. 2A). They included GPCRs (G protein-coupled receptor), with 30 highly up-regulated transcripts encoding for different GPCRs. Interestingly, seven out of these transcripts showed almost no expression in the non-motile cells (fgenesh1_pg.9_421, estExt_fgenesh1_pg.C_6_t10488, gm1.13383_g, fgenesh1_pg.8_348, fgenesh1_pg.36_3, fgenesh1_pg.3_318, gm1.10294_g, gm1.4442_g), with levels below 20 Transcripts Per Kilobase Million (TPM) compared to values ranging from 696 to 3702 in the zoospores (Supplementary Table 2). In addition, many transcripts encoding the signaling transduction route were up-regulated, e.g. 14 Ras GTPases as well as several proteins linked to the CAMP signaling pathway (present in the G protein item in Fig. 2A; Supplementary Table 2).

\section{Cytoskeleton and molecular motors}

Thirty transcripts encoding for dynein (present in the intraflagellar transport and flagellar movement items in Fig. 2A), 24 for myosin and more than 35 for kinesin were up-regulated in zoospores (Fig. 2A and Supplementary Table 2). Genes involved in the tubulin-dependent cytoskeleton as well were largely up-regulated, as expected for flagellated cells. These findings, together with the up-regulation of transcripts encoding for proteins involved in the regulation of cellular motors (putative CAP-GLY domain containing linker protein, CLIP (estExt_fgenesh1_kg.C_140042)) and actin cytoskeleton (formins), reveal a highly dynamic cytoplasm. Actin-Myosin transportation are important for the perception of external stimuli in higher plants as well as in microalgae (Duan and Tominaga, 2018).

\section{DNA replication and transcription}

DNA replication was reduced in zoospores (Fig. 2B), with eight down-regulated transcripts encoding for DNA polymerase, five transcripts encoding for DNA topoisomerase and 11 transcripts encoding for histones (Supplementary Table 2). Genes encoding for RNA polymerases, helicases or involved in 
chromatin remodeling were also significantly down-regulated (Fig. 2C), suggesting that DNA

transcription was lowered. Transcripts involved in the translation process were down-regulated as well. These results suggest that zoospores do not divide, as already suggested by the flow-cytometry data.

\section{Nitrogen and amino acid metabolism}

Genes involved in nitrogen and amino acid metabolisms were highly differentially expressed (Fig. 2D). About 20 genes involved in nitrate and ammonium transport and nitrogen assimilation (such as nitrate (fgenesh1_pm.38_1) and nitrite reductases (e_gw1.38.18.1) or glutamate (estExt_Genewise1.C_17_t10014) and glutamine synthetases (fgenesh1_pm.1_555, fgenesh1_kg.1_553_isotig07286, estExt_Genewise1.C_17_t10014e_gw1.5.701.1)) were strongly upregulated (Supplementary Table 2), possibly denoting the nitrogen deficient medium in which zoospores were produced. In contrast, most of the genes related to the metabolism and turnover of amino acids (amino acid biosynthesis and degradation, urea cycle) were down-regulated. This presumably indicates a reduced need of protein synthesis because of the arrest of the cell division. Genes identified as 'amino acid transport' were predominantly down-regulated, but a few of them were significantly up-regulated with $\log _{2} \mathrm{FC}$ ranging from 4.4 (fgenesh1_pg.18_302) to 1.6 (e_gw1.22.185.1) (Supplementary Table 2). Their function and localization were not further investigated here.

\section{Carbohydrate metabolism}

Genes involved in key steps of the glycolysis (Fig. 2E) were down-regulated, such as triosephosphate isomerase (fgenesh1_kg.10_22_isotig07893), 3-phosphoglycerate kinase enolase (estExt_fgenesh1_kg.C_30041), and pyruvate kinase (estExt_fgenesh1_kg.C_90258) (Supplementary Table 2). The same pattern was observed in several genes coding for the Krebs cycle enzymes, including components of the pyruvate dehydrogenase complex, succinate dehydrogenase (fgenesh1_pm.8_94), citrate synthase (fgenesh1_pg.13_25), isocitrate dehydrogenase (estExt_fgenesh1_kg.C_210136), the T protein of the glycine decarboxylase complex, as well as 
oxidases such as cytochrome c oxidase (fgenesh1_pm.10_34) and alternative oxidase (gw1.27.100.1).

Taken as a whole, these results suggest that carbohydrate catabolism and respiratory activities were down-regulated in zoospores compared with non-motile cells. This assumption was confirmed by the direct measurement of the respiratory activities (Table 2). In R-grown cells, oxygen consumption at D1 considerably augmented, in correspondence to the resuming of cell divisions. Conversely, zoospore respiration slowly declined with time (Table 2).

Fatty acid synthesis and degradation

FAS is involved in the synthesis of 16:0 which can be thereafter elongated and desaturated to produce VLC-PUFAs; PUFA synthase directly produces VLC-PUFAs. Only a few genes involved in the biosynthesis of FAs were differentially expressed in zoospores, at least at D1. The interpretation of the results to decipher how the biosynthetic pathway to VLC-PUFAs is regulated in zoospores is challenging (Fig. 2F). Two desaturases (estExt_fgenesh1_kg.C_1_t10086; gw1.29.176.1) were upregulated whereas e_gw1.11.243.1 was down-regulated, and the only elongases (e_gw1.5.731.1; estExt_fgenesh1_kg.C_60194) found in A. limacinum were down-regulated (Supplementary Table 4). Desaturases and elongases are likely involved in the FAS pathway (for a review, see Morabito et al., 2019), but FAS-1 (e_gw1.21.366.1) was not differentially expressed. Likewise, subunits A and B of the PUFA synthase (PUFA-A fgenesh1_pg.14_\#_251,PUFA-B estExt_fgenesh1_kg.C_140136) were not differentially expressed, and subunit C (PUFA-C estExt_fgenesh1_kg.C_190026) was slightly downregulated (Supplementary Table 4).

FA degradation takes place in both mitochondria and peroxisomes via $\beta$-oxidation. The hydrolysis of FA esterified to glycerolipids is mediated by lipases. About 30 genes were identified as lipase or lipase-like, out of which 17 were up-regulated and 11 down-regulated. Their expression levels showed that lipases were globally upregulated, suggesting that glycerolipid degradation was potentially increased as a whole (Fig. 2F and Supplementary Table 4). Most of the up-regulated lipases belong to class 3 lipases, i.e. involved in the hydrolysis of ester bonds of TAGs or DAGs. However, phospholipases A (Pla1 e_gw1.1.605.1,Pla2 e_gw1.24.47.1), B (Plb e_gw1.6.53.1) and D 
(Pld2 estExt_Genewise1Plus.C_1_t20024, Pld3 gm1.46_g), involved in the degradation of phospholipids, were either non differentially expressed or down-regulated. This suggests that only storage lipids were part of the catabolic processes, whereas membrane-bound glycerolipids are not. FAs released from triacylglycerids (TAGs) are activated as acyl-CoA by acyl-CoA ligases before entering $\beta$-oxidation. An overview of the transcripts involved in FA degradation (Fig. $2 \mathrm{~F}$ ) suggests a global down-regulation. However, a closer inspection indicates that the peroxisomal pathway was up-regulated. Indeed, out of the three genes encoding for Long chain Acyl-CoA ligase, Acs/2 (fgenesh1_kg.4_\#_23_\#_isotig03564) was the only presenting a peroxisomal localization and the only up-regulated one. In addition, most of the transcripts involved in the peroxisomal $\beta$-oxidation (acylCoA ABC transporter; acyl-CoA oxidase Acox1, Acox3; bifunctional enzyme Ehhadh1, Ehhadh3; ketoacyl-CoA thiolase Acaa1, Acaa2, Acaa4) were globally up-regulated (Supplementary Table 4). The mitochondrial $\beta$-oxidation did not show a clear pattern: out of the five genes encoding the acyl-CoA dehydrogenase (Acad), three were down-regulated, one was up-regulated and one did not significantly vary (Supplementary Table 4). Altogether, these results suggest that TAGs are more actively degraded in the zoospores, liberating FAs that are conveyed preferentially to the peroxisome to be oxidized.

Lipid dynamics in zoospores and non-motile cells The expression levels of five genes involved in lipid metabolism (Tagl3 e_gw1.7.228.1, Acox1 fgenesh1_kg.9_\#_301_\#_isotig03404, Acad1 estExt_fgenesh1_kg.C_330018, FAS-1, PUFA-A) were followed for six days in zoospores and non-motile cells (Fig. 3). A $\Delta \Delta C_{T}$ analysis was performed by comparing the expression level of each transcript at day 1 (D1), day 2 (D2), day 4 (D4), and day 6 (D6) with the expression level of the same transcript at D1 in R medium.

The TAG lipase 3 (Tag/3) was strongly up-regulated at D1 in zoospores and represented at this time point the most expressed TAG lipase (Supplementary Table 4). Its expression level did not vary sensibly thereafter (Fig. 3A), suggesting that TAGs were constantly degraded throughout the whole zoospore life. Tagl3 expression in non-motile cells increased progressively to reach a maximal value 
at D4 and D6 (Fig. 3B), i.e. when TAGs accumulate (see below). Highly likely, TAG synthesis and degradation may occur simultaneously in non-motile cells.

$\beta$-oxidation is initiated in peroxisomes and mitochondria through the activities of Acox and Acad, respectively. The peroxisomal Acox1 (Dellero et al., 2018b) and mitochondrial Acad1 (Dellero et al., 2018b) transcripts were the most expressed Acox and Acad in both cell types (Supplementary Table 4). Acox1 was slightly up-regulated in zoospores at D1 and increased thenceforward. Similarly, in non-motile cells, Acox1 expression increased with time, but at a much lower extent than in zoospores. In contrast, the Acad1 expression was down-regulated in zoospores, especially at D6, whereas it did not significantly change in non-motile cells. These results emphasize again the role of peroxisomes vs. mitochondria for the oxidation of FAs in zoospores.

In thraustochytrids, the synthesis of FAs is driven by two enzymatic systems, FAS and PUFA synthase (see Introduction). In zoospores, FAS-1 and PUFA-A genes showed no significant regulation at D1 and D2 compared to non-motile cells (Fig. 3). In the following days, however, their expression decreased in zoospores, especially PUFA-A. In contrast, FAS-1 and PUFA-A expression increased over time in non-motile cells. In both cell types, PUFA-A expression varied significantly more than FAS-1, possibly indicating the higher relevance of the PUFA synthase synthetic pathway in the production of PUFAs. Fatty acid analyses

To correlate gene expression with metabolic changes, the FA content was analyzed during a six-day growth in both $\mathrm{P}$ and $\mathrm{R}$ media (Fig. 4). The major FA species (16:0, 22:5, 22:6) in zoospores increased at D1 (the transfer in P medium occurred on D0) (Fig. 4A), then declined thereafter. In R-grown cells all FA species declined the first day (Fig. 4B) then constantly raised thenceforth to get almost to the initial value at D6. This illustrates the strong relationship between nitrogen availability and lipid metabolism described earlier (Dellero et al., 2018a). In R, cells first consumed their TAGs, then, upon nitrogen limitation, TAGs were synthesized again. In P, nitrogen was limiting right at the beginning of the experiment and TAGs were immediately synthesized until glucose was exhausted, then were consumed to sustain the energy demand. The distribution (\%) of main FAs did not vary much (Figs. 
4C, 4D), although slight differences could be still observed between the two cell populations. Indeed, zoospores showed lower 15:0 and higher 16:0 contents. Odd-numbered FAs (mainly 15:0) are produced from branched-chain amino acids because their degradation leads to propionyl-CoA (Crown et al., 2016) that can replace acetyl-CoA for the synthesis of FAs. In R, the presence of YE, rich in amino acids, corroborates the hypothesis that 15:0 synthesis is driven by the propionyl-CoA derived from branched-chain amino acids. The lack of 15:0 in zoospores might be due to the reduced YE concentration in P medium. Another intriguing difference is the accumulation in zoospores of 18:0 at D1 and 18:1 at D6. The accumulation along the zoospore life span of 18:1 could be due to a residual desaturation activity of 18:0, although no $\Delta 9$-desaturase was found in the genome of $A$. limacinum (Dellero et al., 2018b).

The increase of lipids in P-grown cells at D1 raises the question whether the sporangium or the zoospore is responsible for the recorded lipid accumulation. The release of zoospores and lipid accumulation during the first 24 hours were compared. The release of zoospores started about 8 hours after the transfer in P, and rapidly raised to almost $100 \%$ of the population at 24 hours (Fig. 4E). The FA content in P-grown cells did not vary during the first 12 hours (Fig. 4F), then it nearly doubled at 24 hours. Thus, FAs accumulated after the release of zoospores was initiated, suggesting that they could originate from de novo synthesis.

\section{Discussion}

Zoospores were observed mostly in culture media deprived of amino acids. A nitrogen deficiency cannot explain alone the production of zoospores because replacement of amino acids by $\mathrm{NO}_{3}^{-}$ triggered the release of zoospores during the first days of culture and high biomass accumulation. Amino acids could play a specific role in the life cycle of thraustochytrids. Indeed, thraustochytrids feed on decaying mangrove leaves (Raghukumar et al., 1995), and protein degradation in decaying leaves likely releases amino acids in the close vicinity of the leaf itself. Because nitrogen availability is one of the prime factor limiting cell growth (Alongi, 2009; Rao et al., 1994), the presence or absence of nitrogen-rich organic compounds might be one of the switches controlling the release of 
zoospores from zoosporangia. Oomycete zoospores reach and settle in new territories via chemotaxis, following attractants such as amino acids (Hardham, 2007; Swafford and Oakley, 2017). Similarly, it was demonstrated that zoospores of different thraustochytrid species show a positive chemotactic response to glutamic acid and pectin (Fan et al., 2002). This suggests a specific ability of Aurantiochytrium zoospores to detect the presence of amino acids in the external environment (Dellero et al., 2018a). Indeed, eleven genes involved in amino acid transport were down-regulated and four were up-regulated with fgenesh1_pg.18_302 showing a very low expression level in nonmotile cells. In zoospores its expression strongly increased with a $\log _{2} \mathrm{FC}$ of 4.4 .

The ploidy of $A$. limacinum zoospores and non-motile cells was investigated via two different approaches, namely the distribution of SNPs through the whole exome and flow cytometry. Both methods suggest a diploid status. Flow cytometry experiments showed that the main peak of fluorescence was much sharper in zoospores (Fig. 1E) than in non-motile cells (Fig. 1C), suggesting that zoospores were in $\mathrm{G} 1$ phase and that division/DNA replication was blocked. The latter hypothesis is in agreement with transcriptomic data. Diploidy of $A$. limacinum zoospores corroborates the results reported for $A$. acetophilum for which sexual reproduction has been described (Ganuza et al., 2019). In A. acetophilum biflagellated cells produced by specialized sporangia (Type II sporangia) can act as gametes. The physico-chemical as well as physiological conditions triggering sexual reproduction were not elucidated and the ploidy level of such 'putative gametes' was not tested (Ganuza et al., 2019).

Differential gene expression analyses indicate a profound reprogramming of in zoospores. The transcriptomic data were summarized into a simplified scheme representing some aspects of the metabolism (Fig. 5). Zoospores are potentially characterized by their motility, highly active signal transduction, a low DNA replication activity, a high nitrogen transport activity but a low amino acid metabolism, a low carbohydrate catabolism, high lipase activities and a high $\beta$-oxidation of FAs in peroxisomes. 
G proteins were markedly up-regulated. They are often associated with membrane-spanning receptors (Weis and Kobilka, 2018), and it is possible that the increase of expression level connotes the pioneering activity of zoospores. Indeed, zoospores are likely released to explore new territories, implying a high sensitivity to probe their environment in order to find new substrates or leaves. $G$ proteins also regulate, among others, transcription, cellular differentiation, secretion and motility (Neves et al., 2002). As expected for flagellated cells, most of the genes involved in the cytoskeleton dynamics were strongly up-regulated. The signaling pathway of GPCRs is spread through the whole living kingdom (de Mendoza et al., 2014), e.g. it has been demonstrated that diatoms sense the presence of predators via the GPCR signal transduction pathway (Amato et al., 2018) and that in the rhizarian protist Plasmodiophora brassicae GPCR signal transduction pathways underwent a robust expansion and plays a pivotal role in the germination of resting spores (Bi K. et al., 2019).

Furthermore, in oomycetes a very peculiar GPCR system evolved (Meijer and Govers, 2006).

Conversely, genes involved in DNA replication (such as polymerase, topoisomerase, replication factor etc.) were markedly down-regulated, suggesting that zoospores were blocked in the G1 phase. The arrest of the cell division was presumably associated with a decrease of several metabolic activities. Noteworthy, the amino acid metabolism was largely down-regulated, indicating a potential decline of protein synthesis and turnover. This is true for carbohydrate catabolism as well, since many genes involved in the glycolysis and the Krebs cycle showed a decreased expression in zoospores. Carbohydrate catabolism is strongly connected to the energy metabolism. Thus, results presented here indicate that zoospores have a lower energy demand than dividing cells, despite their swimming activity, an assumption supported by the direct recording of their respiratory activities.

The differential expression of genes involved in lipid metabolism did not show a clear tendency at D1, except for lipase activities, which was up-regulated. In zoospores, the time point D1 corresponded to a metabolic turn, where the FA metabolism shifted from synthesis to catabolism. This may explain the difficulty to correlate gene expressions and FA analyses. Nevertheless, following the expressions of key genes for either FA synthesis or degradation during the whole growth experiment, gene 
expressions and FA analyses correlated well. The gene expression of PUFA-A always varied more strongly than FAS-1 although $22: 6$ and $16: 0$, their respective products, did not. A very fast turnover of either the transcripts or the proteins may be reason of such observation. Indeed, the level of transcripts of the PUFA-A (fgenesh1_pg.14_251) in non-motile cells at D1 (about 56000 TPM) was one of the highest compared to other genes involved in lipid synthesis. A 32-fold increase in expression $\left(\log _{2} \mathrm{FC}=5\right)$ at D6 in non-motile cells was observed, strongly suggesting that a very high number of transcripts is required to sustain a high enzymatic activity. In zoospores, FA catabolism was associated with an increased expression of the the peroxisomal $\beta$-oxidation genes, whilst the mitochondrial $\beta$-oxidation did not follow the same pattern. In many (but not all) organisms, peroxisomes oxidize very long FA chains down to a certain chain length (Reddy and Hashimoto, 2001). The products of the peroxisomal $\beta$-oxidation are then shuttled to mitochondria for a complete oxidation to $\mathrm{CO}_{2}$ and $\mathrm{H}_{2} \mathrm{O}$ (Wanders et al., 2016). In thraustochytrids, 16:0 $\beta$-oxidation would be initiated in peroxisomes because the $16: 0$ and 22:6 content (in percentage, Fig. $4 \mathrm{C}$ ) in zoospores declined at comparable pace.

\section{Potential differences between oomycete and thraustochytrid zoospores}

One of the critical points in the transition from the sedentary to the motile state is the synthesis of flagella. In oomycetes, zoosporogenesis is often induced by a cold shock, and flagella are synthesized from basal bodies at the apex of the nucleus (Hardham, 2007). Zoospores are released upon formation, and the whole process takes about one hour (Hyde et al., 1991). A. limacinum zoospores appear eight hours after transfer in P medium (Fig. 4E). Assuming that zoosporogenesis is induced soon after the transfer, zoospore formation and release appear slower in thraustochytrids than in oomycetes, suggesting that different processes could be involved.

Oomycete zoospores can swim for hours or even days (Hardham, 2007; Kagda et al., 2018), like A. limacinum's. Thus, in both organisms, zoospore survival may depend on the endogenous energy stores. Oomycetes supposedly store energy in mycolaminarin or lipids. Mycolaminarin is a $(1,3,1,6)$ $\beta$-glucan polysaccharide that may represent up to $13 \%$ of the dry weight in oomycetes (Du and 
Mullins, 1998; Lee and Mullins, 1994). The lipid content in the oomycete Phytophthora infestans is rather reduced, representing at best $5 \%$ of the dry weight, with TAGs accounting for half of the total (Griffiths et al., 2003). This is a striking difference with A. limacinum where TAGs may represent up to $30 \%$ of the dry weight (Dellero et al., 2018b), suggesting that the energetic metabolisms in these two organisms rely on different pathways. Interestingly, in $P$. infestans, a taurocyamine kinase (phosphagen kinase of the creatine kinase family) was found to be highly expressed and targeted towards flagellar axonemes during zoosporogenesis, possibly to fulfill the ATP demand associated with the flagellar movement (Kagda et al., 2018). A BLAST search on A. limacinum genome identified three genes of the creatine kinase family homologous to the $P$. infestans gene (estExt_fgenesh1_kg.C_140197, fgenesh1_kg.3_231_isotig05435, fgenesh1_kg.12_175_isotig10864) and up-regulated in zoospores, suggesting that a similar function may be hypothesized in $A$. limacinum. Recently, a differential expression analysis of the of $P$. infestans zoospores vs. mycelia, the vegetative dividing stage (Ah-Fong et al., 2017) revealed that, like in A. limacinum, signal transduction and flagellar proteins were up-regulated, while many metabolic pathways, including glycolysis, TCA cycle and amino acid metabolism were down-regulated. In contrast with results presented here, oomycete zoospores displayed a down-regulation of the $\beta$-oxidation and higher levels of transcripts involved in DNA replication. Although the pathways that generate energy are probably different, it is surprising that DNA replication could increase in non-dividing cells. It was postulated that the developmental program of $P$. infestans zoospores anticipates the need to resume replication after encystment and maturation (Ah-Fong et al., 2017). This was obviously not the case for $A$. limacinum zoospores.

Taken as a whole, the present work shows for the first time that $A$. limacinum zoospores are mainly diploid and that zoosporulation is a complex event depending on external factors, among which the lack of amino acids could play an important role. Here we highlight how the transcriptional reprogramming that occurs in $A$. limacinum zoospores, contributes to the metabolic changes required to migrate, probe their environment and survive; three essential skills to find new territories 
428 and propagate the population. The transcriptional signature of the zoospore reveals the main role

429 played by the G-protein signal transduction pathway in perceiving the external environment,

430 although the actual mediators are still unknown. Lipid metabolism plays an essential role in providing

431 the energy required to swimming for several days and the exploration of new areas.

432 


\section{Experimental procedures}

Strain and media

CCAP 4062/1 strain was collected in Mayotte island (Indian Ocean, $12^{\circ} 48^{\prime} 51.8^{\prime \prime} \mathrm{S}, 45^{\circ} 14^{\prime} 21.7^{\prime \prime} \mathrm{E}$ ) and routinely cultivated at $20^{\circ} \mathrm{C}$ in $250 \mathrm{ml}$ Pyrex ${ }^{\circledR}$ Erlen-Meyer flasks filled with $50 \mathrm{~mL}$ of $\mathrm{R}$ medium (Supplementary Table 1) (Dellero et al., 2018b) with 100 rpm orbital shaking. For all experiments, six day-old axenic cultures grown on $\mathrm{R}$ were transferred to fresh culture media at an initial cell concentration of $5 \times 10^{5}$ cells $\cdot \mathrm{mL}^{-1}$.

The $\mathrm{R}$ and $\mathrm{P}$ media used in the experiments presented here were prepared as described in Dellero et al. (2018a, b); the R2 and R3 recipes are reported in Supplementary Table 1, along with the recipes of R and P. All the culture media were prepared using autoclaved MilliQ water.

Cell enumeration was performed with a Malassez hemocytometer (ca. 100 to 200 cells counted per sample) under a Zeiss AxioScopeA1 (Carl Zeiss SAS, Oberkochen, Germany) microscope. For zoospore enumeration, a sample of $2 \mathrm{~mL}$ was fixed with a drop of $2.5 \%$ glutaraldehyde to immobilize zoospores. All the experiments were run in triplicate.

\section{RNA extraction and sequencing}

For RNA-seq experiments, a six-day-old culture was inoculated in triplicate in $50 \mathrm{~mL}$ of either $\mathrm{R}$ or $\mathrm{P}$ media, at an initial cell concentration of $5 \times 10^{5}$ cells $\cdot \mathrm{mL}^{-1}$. After 24 hours $1.5 \times 10^{7}$ cells were harvested by centrifugation, snap frozen in liquid nitrogen and then stored at $-80^{\circ} \mathrm{C}$ until use. RNA was extracted using the TRI Reagent (Sigma Aldrich) as described by Amato et al. (2017). RNA extracted from biological triplicates of R- and P-grown cultures were sent out for Illumina sequencing. Libraries were produced and processed following the manufacturer's instructions and sequenced on singleend 75 bp mode on NextSeq500 (Illumina, San Diego, CA). The CASAVA 1.8.2 version of the Illumina pipeline was used to process raw data for both format conversion and de-multiplexing.

\section{Bioinformatics analyses of RNA-seq data}

Raw reads were processed with FASTQC

(https://www.bioinformatics.babraham.ac.uk/projects/fastqc/) and BBDuk (https://jgi.doe.gov/data- 
and-tools/bbtools/) in order to check quality and remove low quality bases and adapters. A minimum quality of 25 and a minimum read length of 35 are required. High quality reads were then mapped against the Aurantiochytrium limacinum ATCC ${ }^{\circledast} \mathrm{MYA} 1381^{\mathrm{TM}}$ reference genome (https://genome.jgi.doe.gov/Aurli1/Aurli1.home.html) with STAR (Dobin et al., 2012). FeatureCounts (Liao et al., 2013) was used to perform read summarization at gene level, only reads with quality higher than 30 were used. In addition, strand-specific and paired-end mode are included. Statistical analyses and plots were generated with R software. Lowly expressed genes were filtered out with the HTSFilter package (Rau et al., 2013), then a differential expression analysis was performed using edgeR (Robinson et al., 2009). Genes with an FDR less than or equal to 0.05 were considered significantly differentially expressed. The lists of up- and down-regulated genes were used to perform Gene Ontology Enrichment Analysis (Du et al., 2010) using in-house scripts. The assembled and annotated transcriptome was submitted to NCBI SRA with the accession number PRJNA590015.

Estimation of the heterozygosity level

The mapping files (BAM) obtained by aligning the trimmed RNA-seq reads from the six samples were processed with the Opossum pipeline (Oikkonena and Lise, 2017) in order to remove reads with low quality mapping $(\mathrm{MAPQ}<40)$ and duplicates and to split reads mapping across introns. The three replicates of the R-grown cultures were pooled and the same was done with the three replicates of the P-grown cultures in order to obtain two final BAMs. The final mapping files were then analyzed with Platypus (Rimmer et al., 2014) in order to perform variant calling. Only variants with a depth of at least 10 reads, minimum base quality of 30 , a minimum posterior probability of 30 and classified as PASS were considered. Allele frequencies were calculated as the ratio between the NV and NR fields of the VCF file.

\section{Validation of RNA-seq data and differential gene expression analyses}

qRT-PCR validation of RNA-seq data was carried out on a completely independent experiment as described above for RNA-seq. RNA samples were reverse transcribed using the SuperScript IV VILO Mastermix with ezDNAse kit (ThermoFisher) according to the manufacturer's instructions. Reactions 
were run in a final volume of $10 \mu \mathrm{L}$ containing $10 \mathrm{ng}$ of $\mathrm{CDNA}, 5 \mu \mathrm{L}$ of Power SYBR ${ }^{\circledast}$ Green PCR Master Mix (Applied Biosystems, ThermoFisher) and $600 \mathrm{nM}$ of each primer. Reactions were performed in a CFX ConnectTM Real-Time System (BioRad ${ }^{\circledR}$ ) with the following program: initial denaturation step at $95{ }^{\circ} \mathrm{C}$ for 10 minutes; 40 denaturation-amplification-elongation cycles $\left(95^{\circ} \mathrm{C}, 10 \mathrm{~s} ; 55^{\circ} \mathrm{C}, 10 \mathrm{~s} ; 72{ }^{\circ} \mathrm{C}\right.$, $30 \mathrm{~s}$ ), followed by melting curve assessment $\left(65^{\circ} \mathrm{C}\right.$ to $95^{\circ} \mathrm{C}$, with a $0.5^{\circ} \mathrm{C}$ increment). All primer sequences are available in Supplementary Table 3. Transcript levels were normalized against the geometric mean of three reference genes, with similar expression in both conditions: Pacifastin (estExt_fgenesh1_kg.C_160075), Cystein desulfurase NFS1 (estExt_fgenesh1_kg.C_30063), Protein involved in Snf1 protein kinase complex assembly (gw1.10.847.1) (Supplementary Table 5). For the gene differential expression experiment along a six day-growth, a six-day-old culture grown on $\mathrm{R}$ was inoculated in $50 \mathrm{~mL}$ of either $\mathrm{R}$ or $\mathrm{P}$ fresh culture media at an initial cell concentration of $5 \times 10^{5}$ cells $\cdot \mathrm{mL}^{-1} .1 .5 \mathrm{~mL}$ were gathered by centrifugation at the following time points day 1 (D1), day 2 (D2), day 4 (D4), and day 6 (D6). RNA was extracted and reverse transcribed as described above. qRT-PCR was performed as depicted above. Five genes were analyzed; Tag/3, Acox1, Acad1, FAS-1, PUFA-A. The list of primer sequences is reported in Supplementary Table 5. Gene abbreviations follow Dellero et al. (2018b). The differential expression analyses and statistics were performed using the Pair Wise Fixed Reallocation Randomisation Test method developed in the Relative Expression Software Tool REST@ (Pfaffl et al., 2002). Biological triplicates and technical triplicates of each reaction were performed. Flow cytometry, respiratory activities, lipid extraction and fatty acids analyses To reduce variability, a six-day old R-grown culture was inoculated at $5 \times 10^{5}$ cells $\cdot \mathrm{mL}^{-1}$ in $50 \mathrm{~mL}$ of $P$ medium to induce zoospore formation. After 24 hours, $200 \mu \mathrm{L}$ of the culture were spread onto a $1 \%$ agar-R plate. One of the single colonies was picked and cultivated in R medium for six days. Cells were inoculated in triplicate into $50 \mathrm{~mL}$ of either fresh $\mathrm{R}$ or fresh $\mathrm{P}$ media at a concentration of $5 \times 10^{5}$ cells $\cdot \mathrm{mL}^{-1} . \mathrm{R}$ and $\mathrm{P}$ cultures were harvested at time points 15 hours and 24 hours, corresponding to the production peaks of mononucleated non-motile cells and zoospores, respectively (Dellero et al., 
511 2018a). Three million cells were collected from each sample by centrifugation ( 5 minutes at $3500 \times g$

512 for R cultivated cells and $7000 \times g$ for P cultivated cells). Pellets were washed with $1 \mathrm{~mL}$ of PBS-EDTA 2

$513 \mathrm{mM}$, and then fixed with $500 \mu \mathrm{L}$ of $70 \%$ ethanol for at least 30 minutes at room temperature. After

514 fixation, cells were washed twice with $1 \mathrm{~mL}$ of PBS-EDTA $2 \mathrm{mM}$ and resuspended in $500 \mu \mathrm{L}$ of PBS-

515 EDTA 2 mM. Five microlitres of propidium iodide (PI) and $5 \mu \mathrm{L}$ of RNase I were added and cells were

516 incubated 30 minutes in the dark. All the stained samples were analyzed with a BD FacsCalibur

517 (Benton Dickinson) flow cytometer (excitation $488 \mathrm{~nm}, 585 / 42$ emission filter).

518 Respiration was measured using a Clark-type oxygen electrode (Hansatech, Oxygraph) at $20^{\circ} \mathrm{C}$.

519 Electrode was first calibrated using media saturated with either air or argon for the $100 \%$ and the 0

$520 \%$, respectively. Oxygen consumption was directly monitored in $1 \mathrm{~mL}$ of culture medium.

521 Lipids were extracted according to Folch et al. (1957). FAs were converted into methyl esters (FAME),

522 then analyzed by gas chromatography (GC-MS/FID) on a BPX70 (SGE) column as previously described

523 (Dellero et al., 2018a, b), using 21:0 as internal standard. 
524 Acknowledgments: Morgane Michaud is acknowledged for helpful discussions and informed advice.

525 Véronique Collin-Faure for technical assistance in the flow cytometry experiments. Authors were

526 supported by the French National Research Agency (ANR-10-LABEX-04, GRAL Labex; ANR-11-BTBR-

527 0008, Océanomics; ANR-17-EURE-0003, EUR CBS) and by the Trans'Alg Bpifrance PSPC partnership.

528

529 Conflicts of Interest: The authors declare no conflict of interest.

530

531 


\section{References}

Aasen, I. M., Ertesvåg, H., Heggeset, T. M. B., Liu, B., Brautaset, T., Vadstein, O., et al. (2016)

Thraustochytrids as production organisms for docosahexaenoic acid (DHA), squalene, and carotenoids. App/ Microbiol Biotechnol 100: 4309-4321 (https://doi.org/10.1007/s00253-016-74984).

Agrawal, S. C. (2012) Factors controlling induction of reproduction in algae-review: The text. Folia Microbiol 57: 387-407 (https://doi.org/10.1007/s12223-012-0147-0).

Ah-Fong, A. M. V., Kim, K. S., Judelson, H. S. (2017) RNA-seq of life stages of the oomycete Phytophthora infestans reveals dynamic changes in metabolic, signal transduction, and pathogenesis genes and a major role for calcium signaling in development. BMC Genom 18: 198 (https://doi.org/10.1186/s12864-017-3585-x). Alongi, D. (2009) The energetic of mangrove forests. Dordrecht, Netherlands Springer (Online ISBN 978-1-4020-4271-3) (https://doi.org/10.1007/978-1-4020-4271-3).

Amato, A., Dell'Aquila, G., Musacchia, M., Annunziata, R., Ugarte, A., Maillet, N., et al. (2017) Marine diatoms change their gene expression profile when exposed to microscale turbulence under nutrient replete conditions. Sci Rep 7: 3826 (https://doi.org/10.1038/s41598-017-03741-6). Amato, A., Sabatino, V., Nylund, G. M., Bergkvist, J., Basu, S., Andersson, M. X., et al. (2018) Grazerinduced transcriptomic and metabolomic response of the chain-forming diatom Skeletonema marinoi. ISME J 12: 1594-1604 (https://doi.org/10.1038/s41396-018-0094-0).

Bi, K., Chen, T., He, Z., Gao, Z., Zhao, Y., Liu, H., et al. (2019) Comparative genomics reveals the unique evolutionary status of Plasmodiophora brassicae and the essential role of GPCR signaling pathways. Phytopathol Res 1: 12. (https://doi.org/10.1186/s42483-019-0018-6). Bi, Z.-Q., Ren, L.-J., Hu, X.-C., Sun, X.-M., Zhu, S.-Y., Ji, X.-J., et al. (2018) Transcriptome and gene expression analysis of docosahexaenoic acid producer Schizochytrium sp. under different oxygen supply conditions. Biotechnol Biofuels 11: 249 (https://doi.org/10.1186/s13068-018-1250-5). 
557 Cavalier-Smith, T., Allsopp, M. T. E. P., Chao E. E. (1994) Thraustochytrids are chromists, not fungi:

558 18S rRNA signatures of Heterokonta. Phil Trans R Soc B 346: 387-397

559 (https://doi.org/10.1098/rstb.1994.0156).

560 Chen, X.-R., Xing, Y.-P., Li, Y.-P., Tong, Y.-H., Xu, J.-Y. (2013) RNA-seq reveals infection-related gene

561

562

563

564

565

566 expression changes in Phytophthora capsici. PLOS One 8: e74588 (https://doi.org/10.1371/journal.pone.0074588).

Chen, X.-R., Zhang, B.-Y., Xing, Y.-P., Li, Q.-Y., Li, Y.-P., Tong, Y.-H. et al. (2014) Transcriptomic analysis of the phytopathogenic oomycete Phytophthora cactorum provides insights into infection-related effectors. BMC Genom 15: 980 (https://doi.org/10.1186/1471-2164-15-980).

Crown, S. B., Marze, N., Antoniewicz, M. R. (2016) Catabolism of branched chain amino acids contributes significantly to synthesis of odd-chain and even-chain fatty acids in 3T3-L1 adipocytes. PLOS One 10: e0145850 (https://doi.org/10.1371/journal.pone.0145850).

Dellero, Y., Rose, S., Metton, C., Morabito, C., Lupette, J., Jouhet, J., et al. (2018a) Ecophysiology and lipid dynamics of a eukaryotic mangrove decomposer. Environ Microbiol 20: 3057-3068 (https://doi.org/10.1111/1462-2920.14346).

Dellero, Y., Cagnac, O., Rose, S., Seddiki, K., Cussac, M., Morabito, C. et al. (2018b) Proposal of a new thraustochytrid genus Hondaea gen. nov. and comparison of its lipid dynamics with the closely related pseudo-cryptic genus Aurantiochytrium. Algal Res 35: 125-141 (https://doi.org/10.1016/j.algal.2018.08.018). de Mendoza, A., Sebé-Pedrós, A., Ruiz-Trillo, I. (2014) The evolution of the GPCR signaling system in eukaryotes: modularity, conservation, and the transition to metazoan multicellularity. Genome Biol Evol 6:606 - 619 (https://doi.org/10.1093/gbe/evu038).

Demopoulos, A. W. J., Fry, B., Smith, C. R. (2007) Food web structure in exotic and native mangroves: A Hawaii-Puerto Rico comparison. Oecologia 153: 675-686 (https://doi.org/10.1007/s00442-0070751-x) 
582

583

584

585

586

587

588

589

590

591

592

593

594

595

596

597

598

599

600

601

602

603

604

605

606

Dobin, A., Davis, C. A., Schlesinger, F., Drenkow, J., Zaleski, C., Jha, S., et al. (2012) STAR: Ultrafast universal RNA-seq aligner. Bioinformatics 29: 15-21 (https://doi.org/10.1093/bioinformatics/bts635). Du, X., Mullins, J. T. (1998) Ca2+-induced sporulation in Achlya bisexualis: Reserve 1,3-beta-glucans provide both carbon and phosphorus. Mycologia 90: 990-994

(https://www.jstor.org/stable/3761271).

Du, Z., Zhou, X., Ling, Y., Zhang, Z., Su, Z. (2010) AgriGO: A GO analysis toolkit for the agricultural community. Nucleic Acids Res 38: W64-W70 (10.1093/nar/gkq310).

Duan, Z., Tominaga, M. (2018) Actin-myosin XI: An intracellular control network in plants. Biochem Biophys Res Commun 506: 403-408 (https://doi.org/10.1016/j.bbrc.2017.12.169).

Fan, K. W., Vrijmoed, L. L., and Jones, E. B. (2002) Zoospore chemotaxis of mangrove thraustochytrids from Hong Kong. Mycologia 94: 569-578.

Folch, J., Lees, M. and Sloane-Stanley, G. A. (1957) A simple method for the isolation and purification of total lipids from animal tissues. J Biol Chem 226: 497-509

(http://www.jbc.org/content/226/1/497.long).

Ganuza, E., Yang, S., Amezquita, M., Giraldo-Silva, A., Andersen, R. A. (2019) Genomics, biology and phylogeny Aurantiochytrium acetophilum sp. nov. (Thraustrochytriaceae), including first evidence of sexual reproduction. Protist 170: 209-232 (https://doi.org/10.1016/j.protis.2019.02.004).

Griffiths, R. G., Dancer, J., O'Neill, E., Harwood, J. L. (2003) Effect of culture conditions on the lipid composition of Phytophthora infestans. New Phytol 158: 337-344 (https://doi.org/10.1046/j.14698137.2003.00738.x).

Hardham, A.R. (2007) Cell biology of plant-oomycete interactions. Cell Microbiol 9: 31-39 (https://doi.org/10.1111/j.1462-5822.2006.00833.x).

Honda, D., Yokochi, T., Nakahara, T., Erata, M., Higashihara, T. (1998) Schizochytrium limacinum sp. nov., a new thraustochytrid from a mangrove area in the west pacific ocean. Mycological Res 102: 439-448 (https://doi.org/10.1017/\$0953756297005170). 
Hyde, G. J., Gubler, F., Hardham, A. R. (1991) Ultrastructure of zoosporogenesis in Phytophthora cinnamomi. Mycological Res 95: 577-591 (https://doi.org/10.1016/S0953-7562(09)80072-5).

Isa, H. M., Kamal, A. H. M, Idris, M. H., Rosli, Z., Ismail, J. (2017) Biomass and habitat characteristics of epiphytic macroalgae in the Sibuti mangroves, Sarawak, Malaysia. Tropical Life Sci Res 28: 1-21 (https://doi.org/10.21315/tlsr2017.28.1.1).

Iwasaka, H., Koyanagi, R., Satoh, R., Nagano, A., Watanabe, K., Hisata, K., et al. (2018) A possible trifunctional $\beta$-carotene synthase gene identified in the draft genome of Aurantiochytrium sp. Strain KH105. Genes 9: 200 (https://doi.org/10.3390/genes9040200).

Iwata, I., Kimura, K., Tomaru, Y., Motomura, T., Koike, K., Honda, D. (2017) Bothrosome formation in Schizochytrium aggregatum (Labyrinthulomycetes, Stramenopiles) during zoospore settlement.

Protist 168: 206-219 (https://doi.org/10.1016/j.protis.2016.12.002).

Judelson, H. S., Ah-Fong, A. M. V., Aux, G., Avrova, A. O., Bruce, C., Calkir, C., et al. (2008) Gene expression profiling during asexual development of the late blight pathogen Phytophthora infestans reveals a highly dynamic transcriptome. Mol Plant Microbe Interact 21: 433-447 (https://doi.org/10.1094/MPMI-21-4-0433).

Kagami, M., Von Elert, E., Ibelings, B. W., de Bruin, A., and Van Donk, E. (2007). The parasitic chytrid, Zygorhizidium, facilitates the growth of the cladoceran zooplankter, Daphnia, in cultures of the inedible alga, Asterionella. Proc R Soc B Biol Sci 274: 1561-1566 (doi: 10.1098/rspb.2007.0425). Kagami, M., Miki, T., Takimoto, G. (2014) Mycoloop: chytrids in aquatic food webs. Front Microbio/ 5: 166.

Kagda, M. S., Vu, A. L., Ah-Fong, A. M. V., Judelson, H. S. (2018) Phosphagen kinase function in flagellated spores of the oomycete Phytophthora infestans integrates transcriptional regulation, metabolic dynamics and protein retargeting. Mol Microbiol 110: 296-308 (https://doi.org/10.1111/mmi.14108).

Lee, J. H., Mullins, J. T. (1994) Cytoplasmic water-soluble beta-glucans in achlya-response to nutrient limitation. Mycologia 86: 235-241 (https://www.jstor.org/stable/i290130). 
633 Liang, Y., Liu, Y., Tang, J., Ma, J., Cheng, J., Daroch, M. (2018) Transcriptomic profiling and gene 634 disruption revealed that two genes related to PUFAs/DHA biosynthesis may be essential for cell 635 growth of Aurantiochytrium sp. Mar Drugs 16: 310 (https://doi.org/10.3390/md16090310).

636 Liao, Y., Smyth, G.K., Shi, W. (2013) FeatureCounts: An efficient general purpose program for assigning sequence reads to genomic features. Bioinformatics 30: 923-930. Liu, B., Ertesvåg, H., Aasen, I. M., Vadstein, O., Brautaset, T., Heggeset, T. M. B. (2016) Draft genome sequence of the docosahexaenoic acid producing thraustochytrid Aurantiochytrium sp. T66. Genom Data 8: 115-116 (https://doi.org/10.1016/j.gdata.2016.04.013). of DHA producer Aurantiochytrium under low temperature conditions. Sci Rep 5: 14446 (https://doi.org/10.1038/srep14446). (https://doi.org/10.1094/MPMI-19-1337). metabolism in thraustochytrids. Prog Lipid Res 76: 101007 (https://doi.org/10.1016/j.plipres.2019.101007). 
Pfaffl, M. W., Horgan, G. W., Dempfle, L. (2002) Relative expression software tool (RESTC) for groupwise comparison and statistical analysis of relative expression results in real-time PCR. Nucleic Acids Res 30: e36.

Raghukumar, S. (2008) Thraustochytrid marine protists: Production of PUFAs and other emerging technologies. Mar Biotechnol 10: 631-640 (https://doi.org/10.1007/s10126-008-9135-4). Raghukumar, S., Sathepathak, V., Sharma, S., Raghukumar, C. (1995) Thraustochytrid and fungal component of marine detritus. III. Field studies on decomposition of leaves of the mangrove Rhizophora apiculata. Aquat Microb Ecol 9: 117-125 (https://doi.org/10.1016/0022-0981(94)90160$0)$.

Raghukumar, S., Sharma, S., Raghukumar, C., Sathepathak, V., Chandramohan, D. (1994) Thraustochytrid and fungal component of marine detritus . IV. Laboratory studies on decomposition of leaves of the mangrove Rhizophora apiculata blume. J Exp Mar Biol Ecol 183: 113-131 (https://doi.org/10.1016/0022-0981(94)90160-0).

Rao, R. G., Woitchik, A. F., Goeyens, L., van Riet, A., Kazungu, J., Dehairs, F. (1994) Carbon, nitrogen contents and stable carbon isotope abundance in mangrove leaves from an east african coastal lagoon (Kenya). Aquat Bot 47: 175-183 (https://doi.org/10.1016/0304-3770(94)90012-4). Rau, A., Gallopin, M., Celeux, G., Jaffrézic, F. (2013) Data-based filtering for replicated highthroughput transcriptome sequencing experiments. Bioinformatics 29: 2146-2152.

Reddy, J. K., Hashimoto, T. (2001) Peroxisomal $\beta$-oxidation and peroxisome proliferator-activated receptor $\alpha$ : an adaptive metabolic system. Annu Rev Nutr 21: 193-230 (https://doi.org/10.1146/annurev.nutr.21.1.193).

Rimmer, A., Phan, H., Mathieson, I., Iqbal, Z., Twigg, S. R. F., WGS500 Consortium, et al. (2014) Integrating mapping-, assembly- and haplotype-based approaches for calling variants in clinical sequencing applications. Nat Genet 46: 912-918 (doi:10.1038/ng.3036).

Robinson, M. D., McCarthy, D. J., Smyth, G. K. (2009) Edger: A bioconductor package for differential expression analysis of digital gene expression data. Bioinformatics 26: 139-140. 
683

684

685

686

687

688

689

690

691

692

693

694

695

696

697

698

699

700

701

702

703

704

705

706

707

708

Seddiki, K., Godart, F., Aiese Cigliano, R., Sanseverino, W., Barakat, M., Ortet, P., et al. (2018)

Sequencing, de novo assembly, and annotation of the complete genome of a new thraustochytrid species, strain CCAP_4062/3. Genome Announc 6: e01335-17

(https://doi.org/10.1128/genomeA.01335-17).

Simopoulos, A. P. (2008) The importance of the omega-6/omega-3 fatty acid ratio in cardiovascular disease and other chronic diseases. Exp Biol Med 233: 674-688 (https://doi.org/10.3181\%2F0711MR-311).

Sun, J., Gao, Z., Zhang, X., Zou, X., Cao, L., Wang, J. (2017) Transcriptome analysis of Phytophthora litchii reveals pathogenicity arsenals and confirms taxonomic status. PLOS One 12: e0178245 (https://doi.org/10.1371/journal.pone.0178245).

Suzaki, E., Suzaki, T., Jackson, S. L., Hardham, A. R. (1996) Changes in intracellular pH during zoosporogenesis in Phytophthora cinnamomi. Protoplasma 191: 79-83

(https://doi.org/10.1007/BF01280827).

Swafford, A. J. M., Oakley, T. H. (2017) Multimodal sensorimotor system in unicellular zoospores of a fungus. J Exp Bio/ 221: jeb.163196 (https://doi.org/10.1242/jeb.163196).

Tani, S., Yatzkan, E., Judelson, H. S. (2004) Multiple pathways regulate the induction of genes during zoosporogenesis in Phytophthora infestans. Mol Plant Microbe Interact 17: 330-337

(http://dx.doi.org/10.1094/MPMI.2004.17.3.330).

Taoka, Y., Nagano, N., Okita, Y., Izumida, H., Sugimoto, S., Hayashi, M. (2009) Influences of culture temperature on the growth, lipid content and fatty acid composition of Aurantiochytrium sp. Strain MH0186. Mar Biotechnol 11: 368-374 (https://doi.org/10.1007/s10126-008-9151-4).

Wanders, R. J. A., Waterham, H. R., Ferdinandusse, S. (2016) Metabolic interplay between peroxisomes and other subcellular organelles including mitochondria and the endoplasmic reticulum. Front Cell Dev Bio/ 3: 83 (https://doi.org/10.3389/fcell.2015.00083).

Weis, W. I., Kobilka, B. K. (2018) The molecular basis of G protein-coupled receptor activation. Annu Rev Biochem 87: 897-919 (10.1146/annurev-biochem-060614-033910). 
Figure 1: The ploidy of Aurantiochytrium limacinum cells. (A) Density plot of allele frequencies calculated in non-motile cells and zoospores. Flow cytometry of non-motile cells ( $B$ and $C$ ) and zoospores ( $D$ and $E)$. Light scatter analysis of cell populations showing cell size and granulometry (B and D). Propidium iodide fluorescence ( $\mathrm{C}$ and $\mathrm{E})$.

Figure 2: Differentially expressed genes (cut-off $\log _{2} \mathrm{FC}=|1|$ ) for selected items. (A) signal transduction and cell motility; (B) DNA replication; (C) DNA transcription; (D) nitrogen and amino acid metabolisms; (E) carbohydrate metabolism; (F) lipid metabolism. GPCR: G protein-coupled receptors; RGS: regulator of $G$ protein signaling.

Figure 3: Relative expression of key genes involved in FA synthesis and FA degradation in zoospores (A) and non-motile cells (B). Tag/3 (TAG lipase 3) was chosen as a representative of TAG degradation. FAS-1 and PUFA-A were chosen as representatives of FA synthesis. Acad1 (acyl-CoA dehydrogenase, $\beta$-oxidation in mitochondria) and Acox1 (acyl-CoA oxidase, $\beta$-oxidation in peroxisomes) were chosen for FA degradation. Dotted lines indicate significance threshold. Error bars indicate standard deviation of three independent biological repeats.

Figure 4: FA content in P-grown (red) and R-grown (green) cells. A and B) FA content, expressed as nmoles FA per mg dry weight. C and D) FA distribution, expressed as \% of total FAs. E) Number of zoospores during the first 24 hours expressed as \% of the total cell number. F) FA content evolution during the first 24 hours. Error bars indicate standard deviation of three independent biological repeats.

Figure 5: A schematic representation of Aurantiochytrium limacinum zoospore differential expression analysis based on RNA-seq. Metabolic pathways are illustrated with different colors: pink for DNA replication; purple for FA synthesis; turquoise for elongation and desaturation of FAs; yellow for lipase activities; light orange for signal transduction and $G$ protein pathways; brown for mitochondrial $\beta$-oxidation; grey for peroxisomal $\beta$-oxidation; bright orange for glycolysis and the Krebs cycle; green for nitrogen and ammonium uptake; blue for amino acid metabolism; and dark 
736 blue for flagellar activity and the cytoskeleton dynamics. The colors of full arrows indicate the $\log _{2} \mathrm{FC}$ 737 value (color scale on the right bottom of the figure) for each gene involved in lipid metabolism. The 738 empty arrows indicate the trend of the metabolic pathway. Abbreviations: SFA, saturated FAs; PUFA, 739 polyunsaturated FAs; TAG, triacylglycreol; DAG, diacylglycerol; MAG, monoacylglycerol. Gene 740 abbreviations (in alphabetic order): abcd, peroxisomal ABC transporter; $A C C$, acetyl-CoA carboxylase; 741 Acaa, 3-ketoacyl-CoA thiolase; Acad, acyl-CoA dehydrogenase; Acox, acyl-CoA oxidase; Acsl, long 742 chain acyl-CoA ligase; CACT, carnitine acylcarnitine transferase; CPT, carnitine palmitoyl transferase; $743 \Delta$ elo, fatty acid $\Delta$ elongase; $\Delta F A D, \Delta$ fatty acid desaturase; Dagl, diacylglycerol lipase; Ech, enoyl-CoA 744 hydratase; Ehhadh, bifunctional enzyme; Fas, fatty acid synthase; Hadh, 3-hydroxyacyl-CoA 745 dehydrogenase; HADH, trifunctional enzyme; Magl, monoacylglycerol lipase; $\Omega 3 F A D, \Omega 3$ fatty acid 746 desaturase; Tagl, triacylglycerol lipase. 
Table 1: Effect of the medium composition on the production of zoospores. The initial cell concentration was set at $5 \times 10^{5}$ cells $\mathrm{mL}^{-1}$ in all conditions. $\mathrm{P}^{*}=$ after 48 hours growth, amino acids were added to the culture at the same concentration as R3 (Supplementary Table 1). The number of zoospores is expressed as percentage of total cells at day 1 (D1), day 2 (D2), day 4 (D4) and day 6 (D6). Experiments were run in triplicate.

\begin{tabular}{|c|c|c|c|c|c|}
\hline & \multicolumn{5}{|l|}{ Media } \\
\hline & $\mathbf{R}$ & $\mathbf{P}$ & R2 & R3 & $\mathbf{P}^{*}$ \\
\hline \multirow[t]{2}{*}{ C source } & $6 \%$ & $0.15 \%$ & $6 \%$ & $6 \%$ & $0.15 \%$ \\
\hline & Glucose & Glucose & Glucose & Glucose & Glucose \\
\hline \multirow[t]{2}{*}{$\mathrm{N}$ and $\mathrm{P}$ sources } & $2 \% \mathrm{YE}$ & $0.05 \% \mathrm{YE}$ & $\mathrm{NaNO}_{3}$ & AA & $0.05 \% \mathrm{YE}$ \\
\hline & & & $\mathrm{NaH}_{2} \mathrm{PO}_{4}$ & $\mathrm{NaH}_{2} \mathrm{PO}_{4}$ & $+A A(D 2)$ \\
\hline Zoospores at D1 & $<3 \%$ & $85 \%$ & $92 \%$ & $<4 \%$ & $90 \%$ \\
\hline \multirow[t]{2}{*}{ Zoospores at D2 } & $<1.5 \%$ & $98 \%$ & $83 \%$ & $<2 \%$ & $98 \%$ \\
\hline & & & & & $+\mathrm{AA}$ \\
\hline Zoospores at D4 & $<1.5 \%$ & $96 \%$ & $80 \%$ & 0 & $4.8 \%$ \\
\hline Zoospores at D6 & 0 & $88 \%$ & $25 \%$ & 0 & $3.4 \%$ \\
\hline $\begin{array}{l}\text { Total number of cells } \\
\text { at } \mathrm{D} 6\left(\times 10^{7} \text { cells. } \mathrm{mL}^{-1}\right)\end{array}$ & 7.2 & 0.45 & 10 & 8.9 & 0.36 \\
\hline
\end{tabular}


Table 2: Respiratory activity in R- and P-grown cells along a six-day growth. Oxygen consumption rates are expressed as nmoles $\mathrm{O}_{2} \mathrm{~min}^{-1}$ per million cells \pm SD. Experiments were run in triplicate.

\section{Respiratory activity after transfer to new media}

\begin{tabular}{llllll}
\hline Days & $\mathbf{0}$ & $\mathbf{1}$ & $\mathbf{2}$ & $\mathbf{4}$ & $\mathbf{6}$ \\
\hline R-grown & $1.71 \pm 0.16$ & $6.16 \pm 1.27$ & $2.75 \pm 0.62$ & $3.06 \vee 0.52$ & $2.96 \pm 0.24$ \\
& & & & & \\
\hline P-grown & $1.71 \pm 0.16$ & $1.76 \pm 0.22$ & $0.53 \pm 0.1$ & $0.44 \pm 0.05$ & $0.16 \pm 0.05$ \\
\hline
\end{tabular}



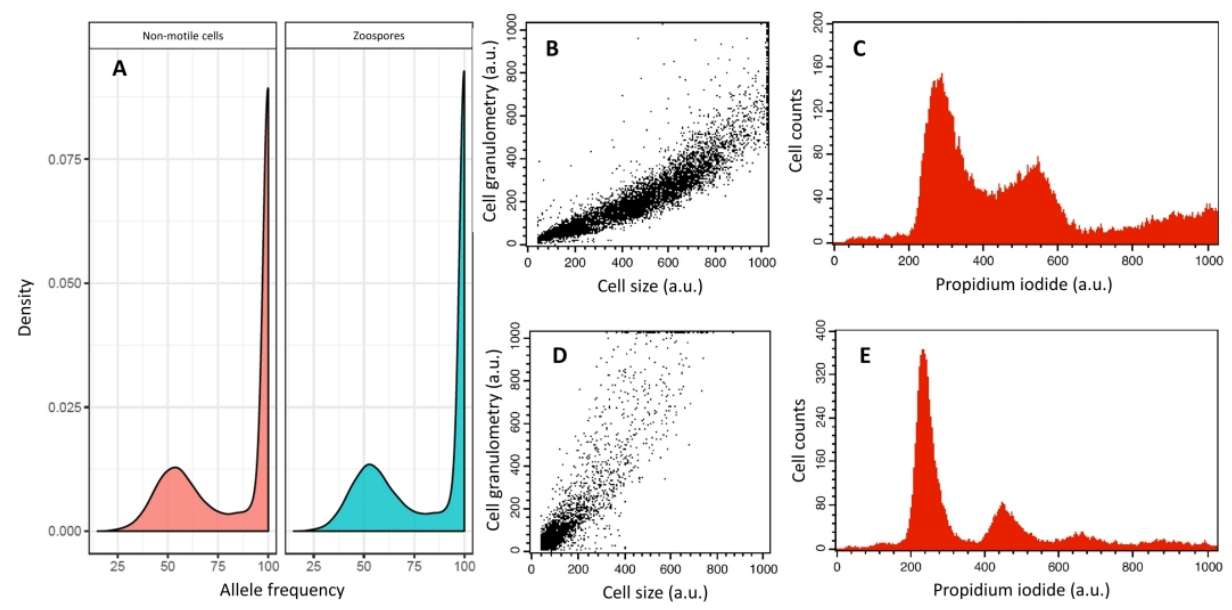

The ploidy of Aurantiochytrium limacinum cells. (A) Density plot of allele frequencies calculated in nonmotile cells and zoospores. Flow cytometry of non-motile cells ( $B$ and $C$ ) and zoospores ( $D$ and $E$ ). Light scatter analysis of cell populations showing cell size and granulometry ( $B$ and $D$ ). Propidium iodide fluorescence ( $\mathrm{C}$ and $\mathrm{E})$.

$338 \times 190 \mathrm{~mm}(300 \times 300 \mathrm{DPI})$ 
A Signal transduction and cell motility

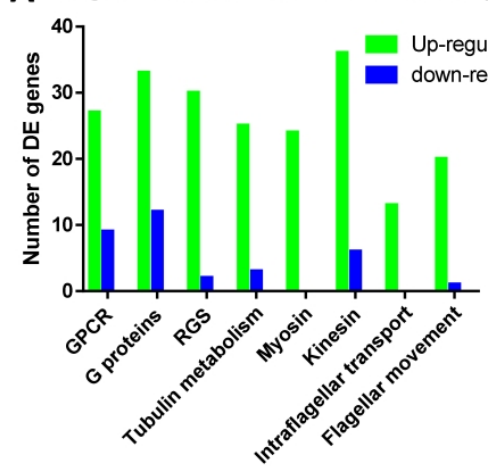

C
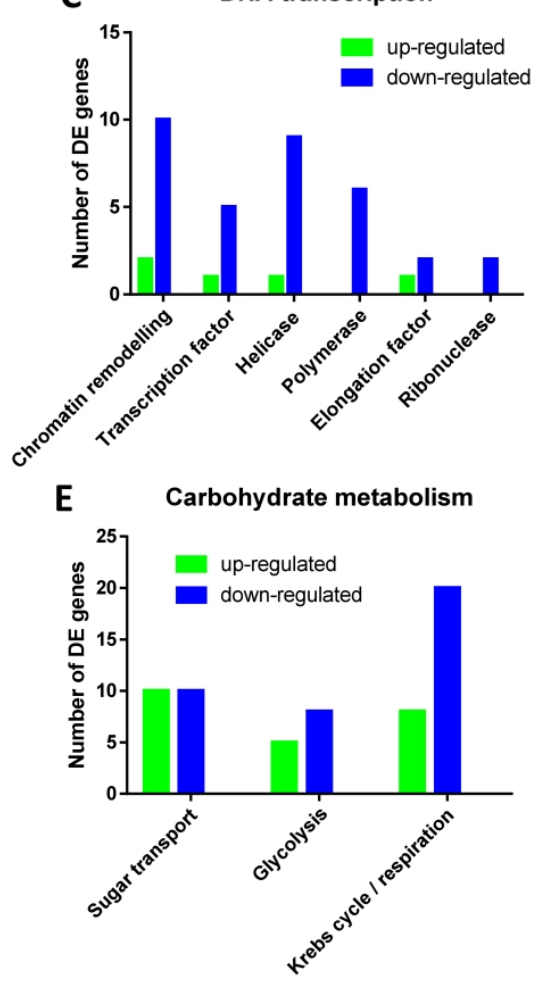

B

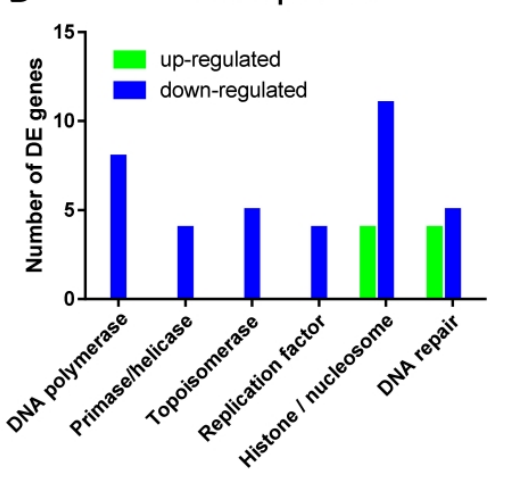

D Nitrogen and amino acid metabolism

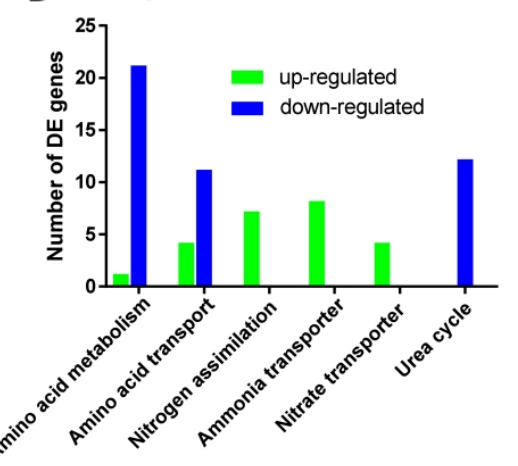

$\mathbf{F}$

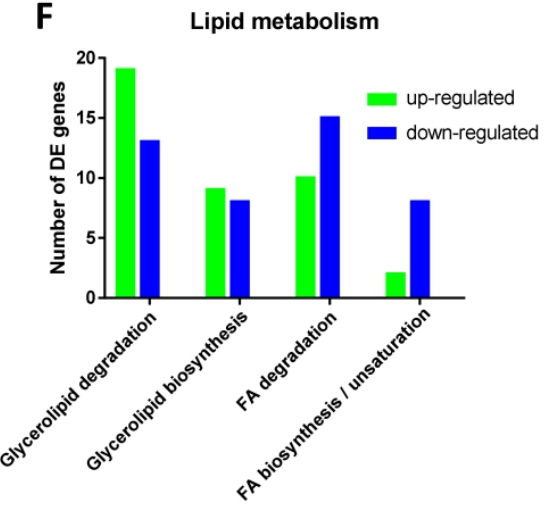

Differentially expressed genes (cut-off Log2FC $=|1|$ ) for selected items. (A) signal transduction and cell motility; (B) DNA replication; (C) DNA transcription; (D) nitrogen and amino acid metabolisms; (E) carbohydrate metabolism; (F) lipid metabolism. GPCR: G protein-coupled receptors; RGS: regulator of G protein signaling. 

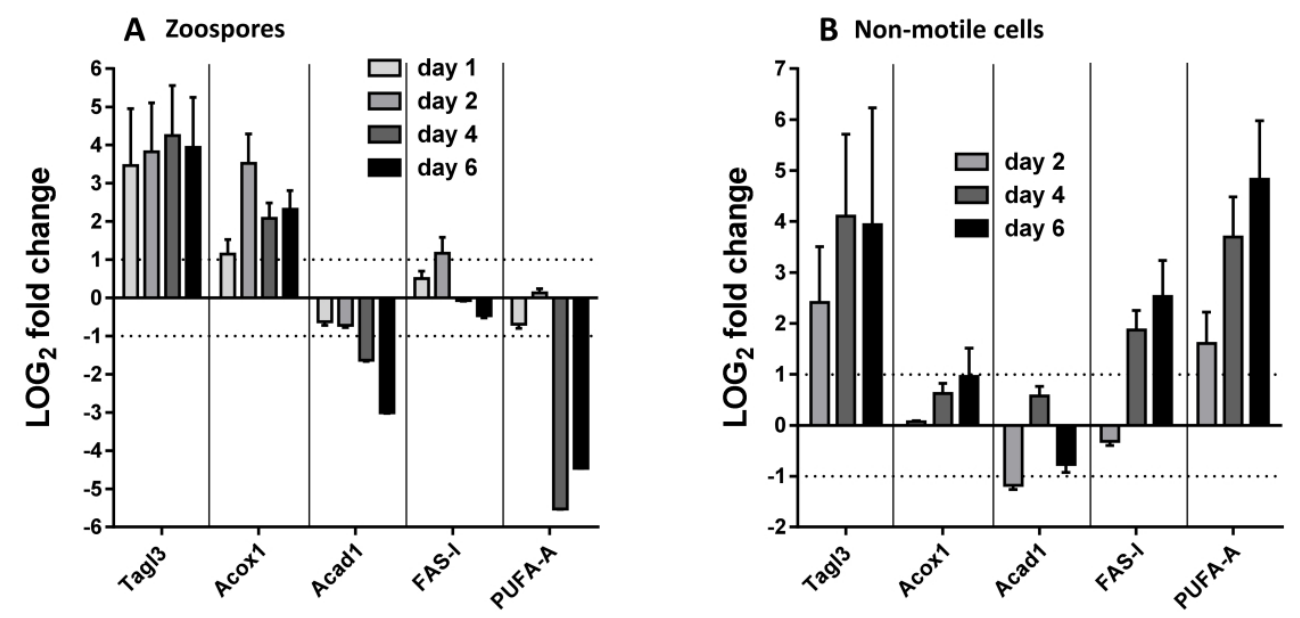

Relative expression of key genes involved in FA synthesis and FA degradation in zoospores (A) and nonmotile cells (B). Tagl3 (TAG lipase 3 ) was chosen as a representative of TAG degradation. FAS- 1 and PUFA-A were chosen as representatives of FA synthesis. Acad1 (acyl-CoA dehydrogenase, $\beta$-oxidation in mitochondria) and Acox1 (acyl-CoA oxidase, $\beta$-oxidation in peroxisomes) were chosen for FA degradation. Dotted lines indicate significance threshold. Error bars indicate standard deviation of three independent biological repeats. 

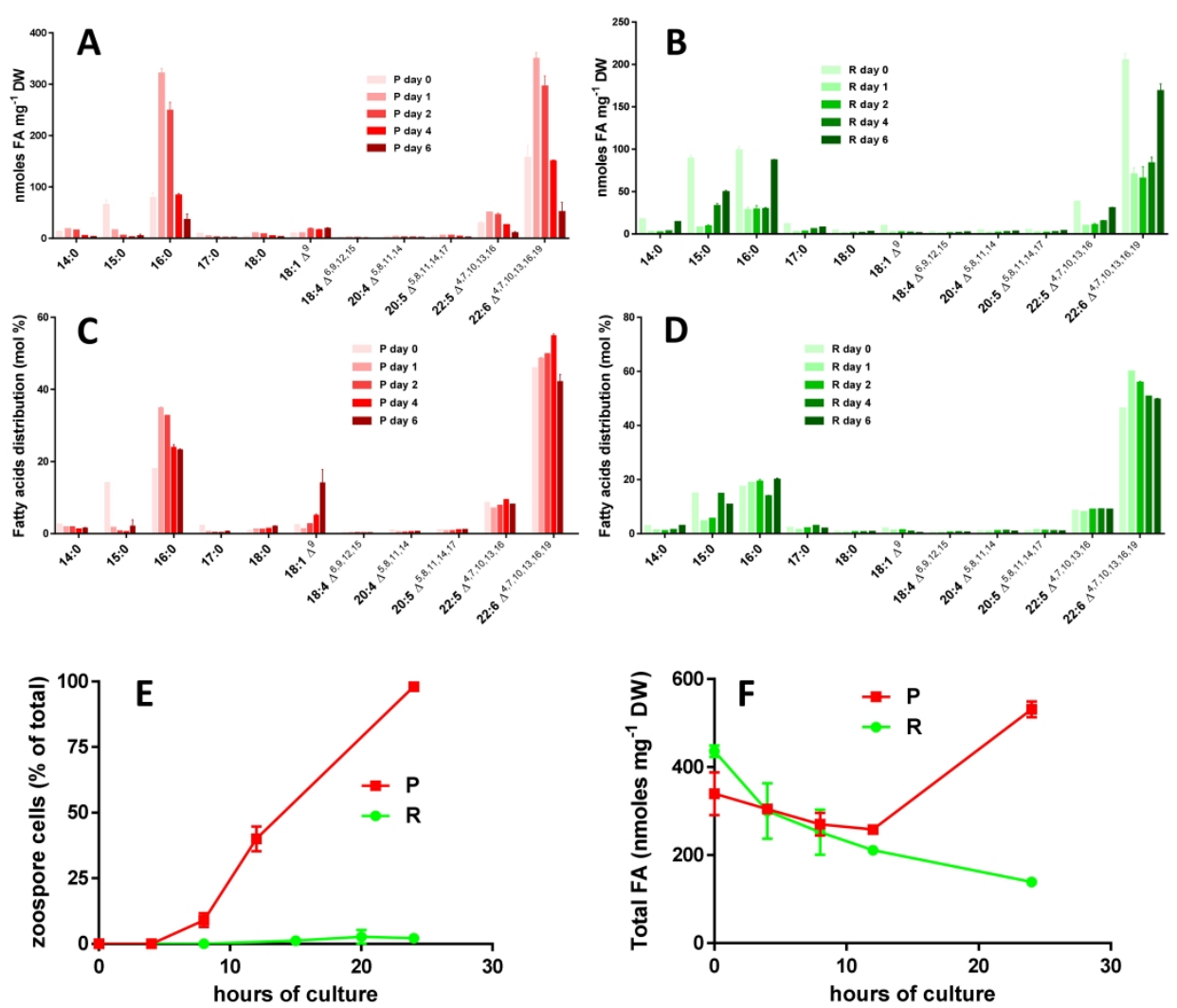

FA content in P-grown (red) and R-grown (green) cells. A and B) FA content, expressed as nmoles FA per mg dry weight. C and D) FA distribution, expressed as \% of total FAs. E) Number of zoospores during the first 24 hours expressed as \% of the total cell number. F) FA content evolution during the first 24 hours.

Error bars indicate standard deviation of three independent biological repeats.

$192 \times 161 \mathrm{~mm}(600 \times 600 \mathrm{DPI})$ 


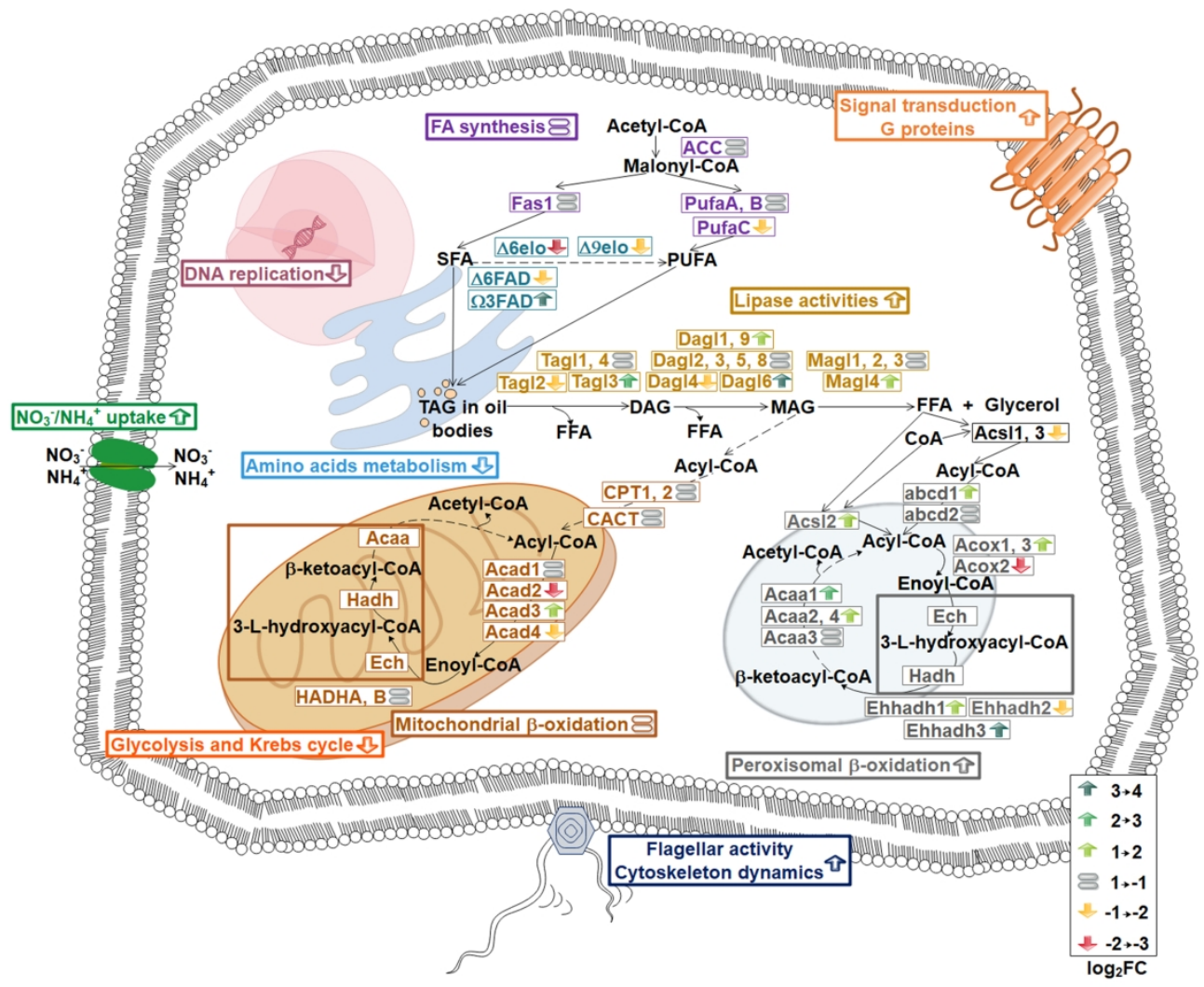

A schematic representation of Aurantiochytrium limacinum zoospore differential expression analysis based on RNA-seq. Metabolic pathways are illustrated with different colors: pink for DNA replication; purple for FA synthesis; turquoise for elongation and desaturation of FAs; yellow for lipase activities; light orange for signal transduction and $\mathrm{G}$ protein pathways; brown for mitochondrial $\beta$-oxidation; grey for peroxisomal $\beta$ oxidation; bright orange for glycolysis and the Krebs cycle; green for nitrogen and ammonium uptake; blue for amino acid metabolism; and dark blue for flagellar activity and the cytoskeleton dynamics. The colors of full arrows indicate the log2FC value (color scale on the right bottom of the figure) for each gene involved in lipid metabolism. The empty arrows indicate the trend of the metabolic pathway. Abbreviations: SFA, saturated FAs; PUFA, polyunsaturated FAs; TAG, triacylglycreol; DAG, diacylglycerol; MAG, monoacylglycerol. Gene abbreviations (in alphabetic order): abcd, peroxisomal ABC transporter; ACC, acetyl-CoA carboxylase; Acaa, 3-ketoacyl-CoA thiolase; Acad, acyl-CoA dehydrogenase; Acox, acyl-CoA oxidase; Acsl, long chain acyl-CoA ligase; CACT, carnitine acylcarnitine transferase; CPT, carnitine palmitoyl transferase; $\triangle$ elo, fatty acid $\Delta$ elongase; $\triangle F A D, \Delta$ fatty acid desaturase; Dagl, diacylglycerol lipase; Ech, enoyl-CoA hydratase; Ehhadh, bifunctional enzyme; Fas, fatty acid synthase; Hadh, 3-hydroxyacyl-CoA dehydrogenase; $\mathrm{HADH}$, trifunctional enzyme; Magl, monoacylglycerol lipase; $\Omega 3 F A D, \Omega 3$ fatty acid desaturase; Tagl, triacylglycerol lipase.

$224 \times 189 \mathrm{~mm}(300 \times 300 \mathrm{DPI})$ 